\title{
The serine/threonine kinase LKB1 controls thymocyte survival through regulation of AMPK activation and $\mathrm{Bcl}-\mathrm{XL}$ expression
}

\author{
Yonghao $\mathrm{Cao}^{1, *}$, Hai $\mathrm{Li}^{1,}$, , Haifeng $\mathrm{Liu}^{1}$, Chao Zheng ${ }^{1}$, Hongbin $\mathrm{Ji}^{1}$, Xiaolong $\mathrm{Liu}^{1}$ \\ ${ }^{1}$ Laboratory of Molecular Cell Biology, Institute of Biochemistry and Cell Biology, Shanghai Institutes for Biological Sciences, \\ Chinese Academy of Sciences, Shanghai 200031, China
}

LKB1 is a serine/threonine kinase that directly activates the energy sensor AMP-activated protein kinase (AMPK) in response to bioenergetic stress, and mainly acts as a tumor suppressor that controls cell polarity and proliferation. Although LKB1 is expressed in multiple tissues including the thymus and the spleen, its roles in T-cell development and function remain unknown. Here, we show that T-cell-specific deletion of LKB1 resulted in reduced survival of double-positive (DP) thymocytes and impaired generation of both CD4 and CD8 single-positive thymocytes. Disruption of LKB1 not only prevented the activation of AMPK but also impaired the expression of anti-apoptotic protein Bcl-XL. Importantly, ectopic expression of either Bcl-XL or the constitutively active AMPK mutant significantly rescued DP thymocytes from LKB1 deficiency-induced cell death. Moreover, ectopic expression of the constitutively active AMPK mutant was found to restore the expression of Bcl-XL in LKB1-deficient DP thymocytes. These findings identify LKB1 as a critical factor for the survival of DP thymocytes through regulation of AMPK activation and BclXL expression.

Keywords: LKB1, AMPK, Bcl-XL, thymocyte, survival, development

Cell Research (2010) 20:99-108. doi: 10.1038/cr.2009.141; published online 22 December 2009

\section{Introduction}

Intrathymic T-cell development is a time-consuming process, and survival is a prerequisite for thymocytes to undergo selection and maturation in the thymus. Therefore, thymocytes must efficiently acquire metabolic substrates from their extracellular environment and actively maintain cellular energy homeostasis at distinct developmental stages $[1,2]$. Numerous studies have suggested that growth factors such as IL-7 and IL-4 promote nutrient uptake in $\mathrm{T}$ cells by increasing the expression of nutrient transporters $[1,2]$, while intracellular molecules PI3K, AKT and TOR play important roles in the regulation of catabolic and anabolic metabolism [1-

\footnotetext{
*These two authors contributed equally to this work. Correspondence: Xiaolong Liu

Tel: +86-21-54921176; Fax: +86-21-54921178

E-mail: liux@sibs.ac.cn

Received 4 November 2009; revised 9 November 2009; accepted 19 November 2009; published online 22 December 2009
}

4]. Specifically, Notch receptor, IL-7 receptor (IL-7R) and pre-T-cell receptor (pre-TCR) are expressed in the double-negative (DN) thymocytes, and signals transduced by these receptors accelerate the nutrient uptake and consequentially promote $\mathrm{DN}$ thymocyte survival [5-8], while TCR signal-induced Bcl-2 expression is essential for the survival of positively selecting thymocytes [9-11]. However, how the intermediate double-positive (DP) thymocytes, which do not express Notch and IL$7 \mathrm{R}$ molecules and whose survival is TCR independent [12-14], actively prolong their life span to complete the $\mathrm{TCR} \alpha($ T $r a)$ recombination and wait for being positively selected remains largely unknown.

The serine/threonine (Ser/Thr) kinase LKB1 (also known as STK11) is the main upstream kinase of the AMP-activated protein kinase (AMPK) cascade that senses cellular energy state and adjusts cellular metabolism [15-17]. LKB1 directly activates AMPK in response to rising AMP:ATP ratio [16]. Once activated, AMPK phosphorylates several substrates, leading to repression of energy-consuming processes and simultaneous en- 
hancement of energy-generating processes, such as inactivation of mTOR, downregulation of protein synthesis, enhancement of glucose uptake and glycolysis, thereby maintaining cellular energy homeostasis $[15,16,18]$. LKB1 was originally identified as a tumor suppressor that modulates epithelial cell polarity and proliferation $[15,19]$, and its inactivation is a hallmark of Peutz-Jeghers syndrome characterized by hamartomatous proliferation and gastrointestinal polyps [20-23]. Moreover, LKB1 has been shown to participate in regulating early embryonic development [24, 25]. Germline knockout of $L K B 1$ alleles results in embryonic lethality at mid-gestation with multiple developmental abnormalities [25].

Because LKB1 plays an important role in regulating cellular energy balance and is expressed in thymus and spleen [22], we were curious to know whether LKB1 is involved in regulating T-cell development and function. Here, we describe the generation and characterization of mice whose T cells lack LKB1 and show that T-cellspecific deletion of LKB1 resulted in reduced survival of DP thymocytes and impaired generation of both CD4 and CD8 single-positive (SP) thymocytes. Our studies indicate that LKB1 plays a critical role in the regulation of both the activation of energy sensor AMPK and the expression of anti-apoptotic protein Bcl-XL, through which LKB1 controls the survival of DP thymocytes.

\section{Results}

T-cell-specific deletion of LKB1 disrupts thymocyte development

To study the function of LKB1 in T-cell development, mice bearing a LoxP-flanked gene encoding LKB1 $\left(L K B 1^{\mathrm{fl} / \mathrm{f}}\right)$ [20] were bred with mice expressing the Cre recombinase under the control of the proximal $L c k$ promoter ( $L c k C r e)$ [26] to generate $L c k C r e^{+} L K B 1^{\mathrm{fl} / f 1}$ mice (homozygous mutant, thereafter referred to as LKB1deficient). The floxed $L K B 1$ alleles were efficiently deleted in thymocytes, but not in the tails of LKB1deficient mice, as expected (Figure 1A). To follow LKB1 deletion during T-cell development, genomic DNA from sorted thymocyte subsets of LKB1-deficient mice was used to amplify the floxed and deleted alleles. We found that the deletion of floxed alleles started at the DN3 stage and was virtually complete at the DN4 stage (Figure 1B). Accordingly, the $L K B 1 \mathrm{mRNA}$ was detectable in the DN3 cells (about $20 \%$ of control), but not in the DN4 (less than $2 \%$ of control) and DP cells (less than $2 \%$ of control) (Figure 1C). Notably, DP thymocytes from $L c k \mathrm{Cre}^{-} L K B 1^{+/ f l}$ mice (non-deletion mutant, thereafter referred to as 'wild-type') expressed the highest level of LKB1 mRNA compared to other subsets from the same
A

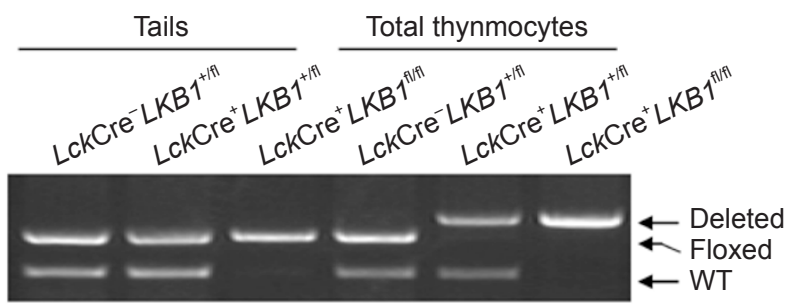

B
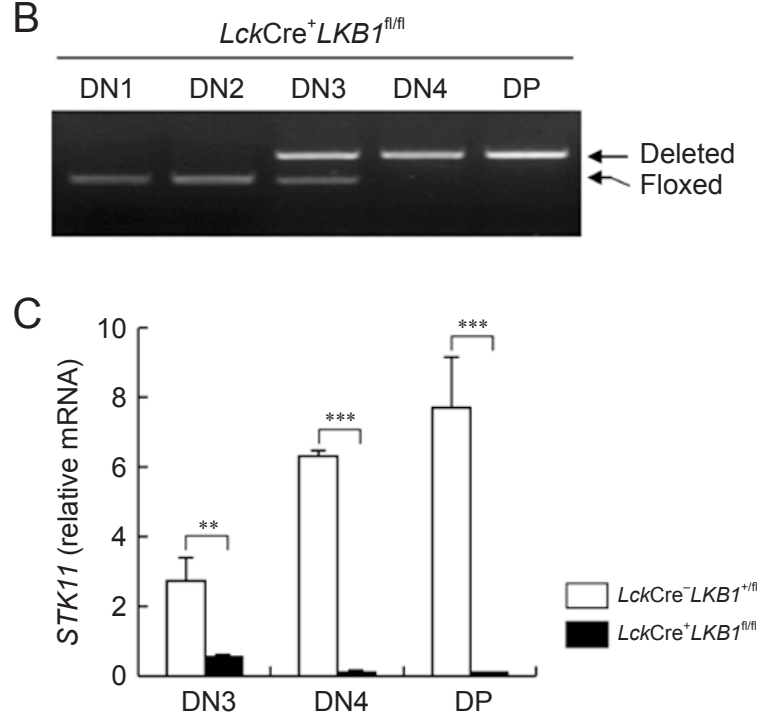

Figure 1 T-cell-specific deletion of $L K B 1$ alleles. (A) Genomic DNA was isolated from either tails or total thymocytes of indicated genotypes and then used for PCR analysis of $L K B 1$ deletion. The wild-type $L K B 1$ allele (WT) was amplified as a 120bp band, the LoxP-flanked allele (Floxed) as a 490-bp band and the deleted allele (Deleted) as a 680-bp band. (B) Genomic DNA from sorted DN1-4 and DP thymocytes was tested for deletion efficiency of the $L K B 1$ locus. (C) Quantification of STK11 (encoding LKB1) mRNA expression. DN3, DN4 and DP thymocytes from LKB1-deficient and control mice were used for realtime RT-PCR analysis. Data are expressed as mean \pm SEM. $* * P<0.01 ; * * * P<0.001$. Data are representative of three (A, B, C) independent experiments.

mice (Figure 1C).

To determine the impact of T-cell-specific deletion of LKB1 on thymocyte development, the thymic cellularity and subset composition of LKB1-deficient mice were analyzed. A fivefold reduction in total thymocyte number was found in the LKB1-deficient mice relative to $L c k \mathrm{Cre}^{-} L K B 1^{+/ f l}$ mice (Figure $2 \mathrm{~A}$ ). Notably, $L c k \mathrm{Cre}^{+} L K B 1^{+/ f l}$ mice (heterozygous mutant) had $15 \%$ fewer total thymocytes than wild-type mice (Figure 2A), suggesting that LKB1 activity is sensitive to gene dosage. Fluorescence-activated cell sorting (FACS) analysis further revealed that LKB1-deficient mice had a marked 

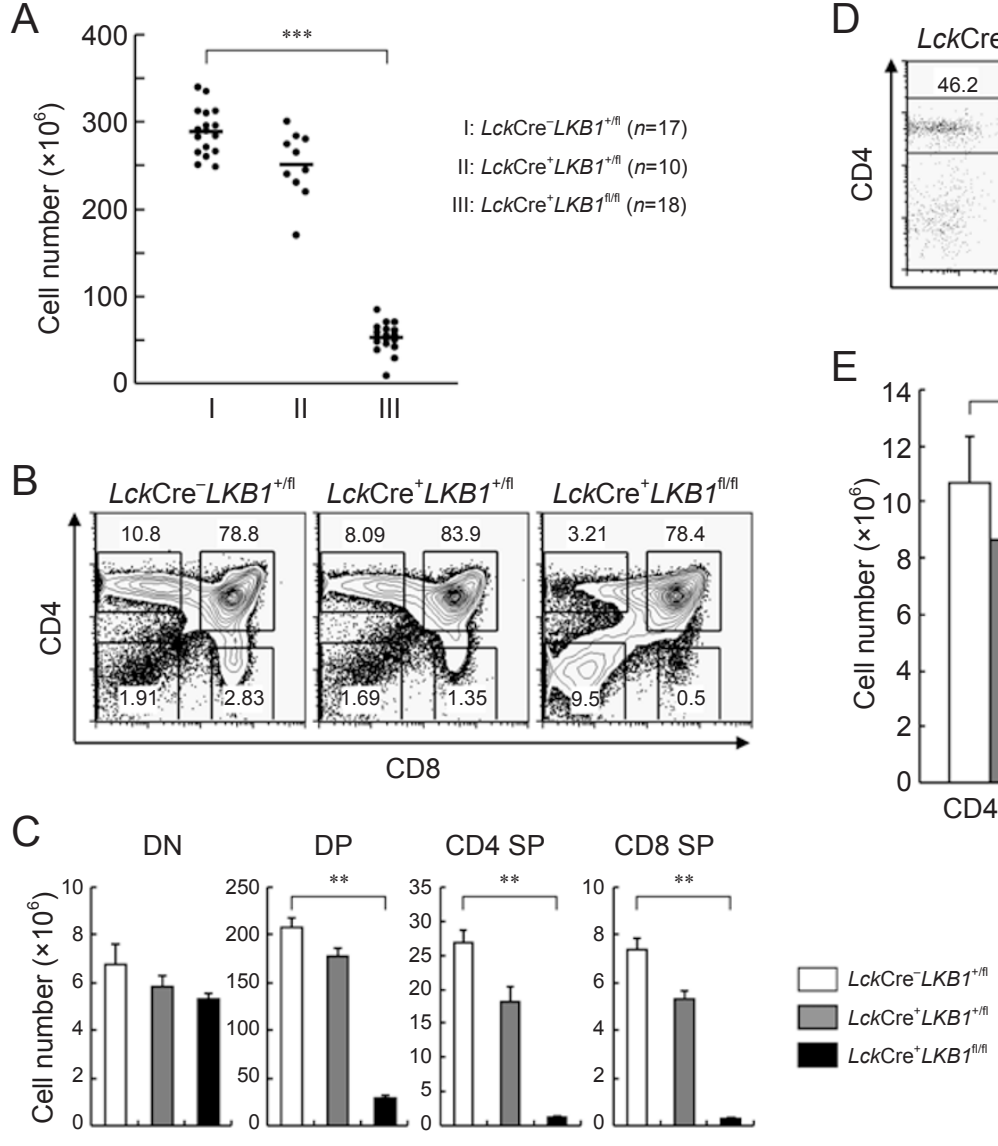
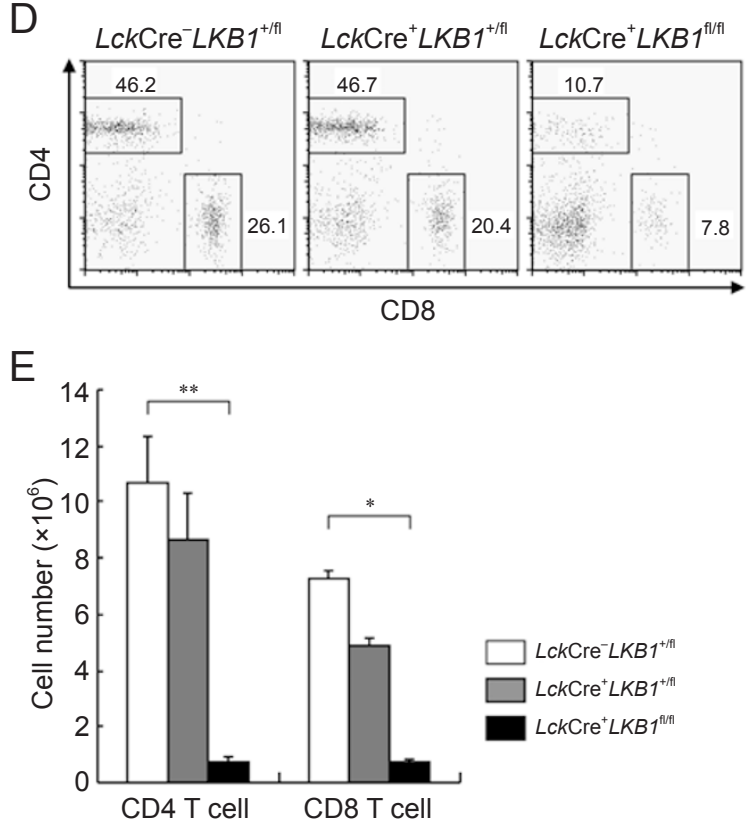

Figure 2 Targeted deletion of LKB1 in thymocytes severely impairs intrathymic T-cell development. (A) Comparison of total thymocyte numbers of age-matched LKB1-deficient $(n=18)$, heterozygous mutant $(n=10)$ and wild-type $(n=17)$ mice. The cell numbers shown are from at least 10 separate experiments. ' $\bullet$ Represents cell number of individual mouse; '-' represents the mean thymocyte number in each group. ${ }^{* * *} P<0.001$. (B) Total thymocytes were analyzed for CD4 and CD8 expression by flow cytometry. Numbers next to or within boxes of contour diagrams indicate the percentage of cells. (C) Cell numbers of thymic subpopulations were quantified by multiplying total live cell numbers by the fraction of cells in that population and presented as mean \pm SEM. $* * P<0.01$. (D) Total lymphocytes were analyzed for CD4 and CD8 expression by flow cytometry. The numbers next to boxes indicate the percentage of cells. (E) The absolute cell numbers of CD4 and CD8 T-cell subsets were quantified by multiplying total cell numbers by the fraction of cells in that population. Data are expressed as mean \pm SEM. $* P<0.05 ; * * P<0.01$. Data are representative of three (C, D, E) or four (B) independent experiments.

reduction in the percentages of $\mathrm{CD} 4 \mathrm{SP}$ and $\mathrm{CD} 8 \mathrm{SP}$ populations, with a corresponding increase in the frequency of DN population (Figure 2B), and the final calculation indicated that the absolute numbers of DP, CD4 SP and CD8 SP, but not DN cells, in LKB1-deficient mice were significantly decreased (Figure $2 \mathrm{C}$ ). Accordingly, the percentages of both CD4 and CD8 T cells from LKB1-deficient mice were notably lower than those of wild-type controls (Figure 2D), and a more than 10-fold reduction in total T-cell number was found in the lymph nodes of LKB1-deficient mice (Figure 2E).

LKB1-deficient mice exhibit a developmental block at the DN3 to DN4 transition
The dramatic reduction in thymic cellularity in LKB1deficient thymus could be due to defects in the development of the early T-cell progenitors within the DN stage [8]. To examine the differentiation capacities of the DN thymocytes in LKB1-deficient mice, we fractionated DN cells by staining for CD44 and CD25 as described [27]. Little change was detected in the distribution of DN14 subsets between wild-type and heterozygous mutant mice (Figure 3A). Intriguingly, LKB1-deficient mice, in which the floxed $L K B 1$ alleles were substantially deleted at the DN3 stage, had more DN3 cells and fewer DN4 cells compared with their wild-type controls (Figure 3A and $3 \mathrm{~B}$ ). The increased DN3/DN4 ratio suggests that the transition from DN3 to DN4 was somewhat impaired in 
LKB1-deficient mice.

One of the critical events during early thymocyte development is TCR $\beta$ locus (Tcrb) rearrangement. Only cells that productively rearranged their Tcrb locus express the TCR $\beta$ chain that assembles with $\mathrm{CD} 3$ and pre-
$\mathrm{T} \alpha$ molecules to form a signaling-competent pre-TCR, allowing their progression to the DN4 stage [8]. Analysis of intracellular TCR $\beta$ expression showed that LKB1deficient DN3 and DN4 cells had similar subpopulations that had substantially expressed intracellular TCR $\beta$
A

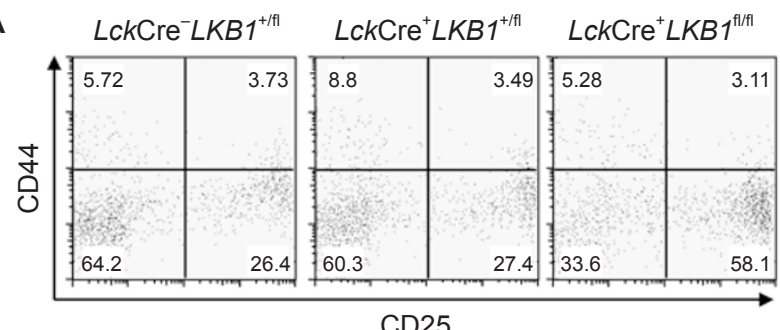

B

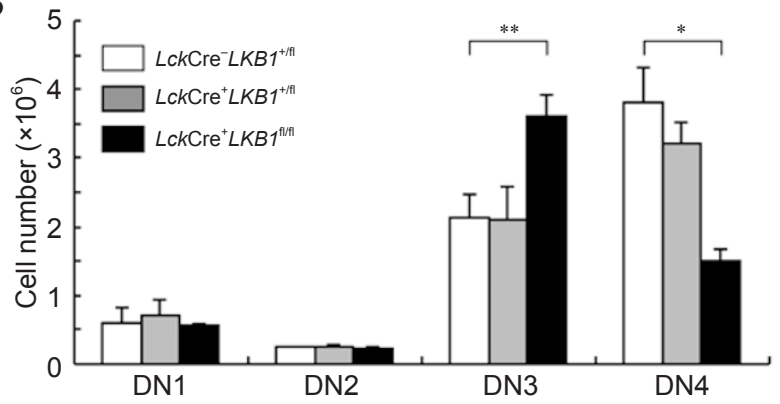

C

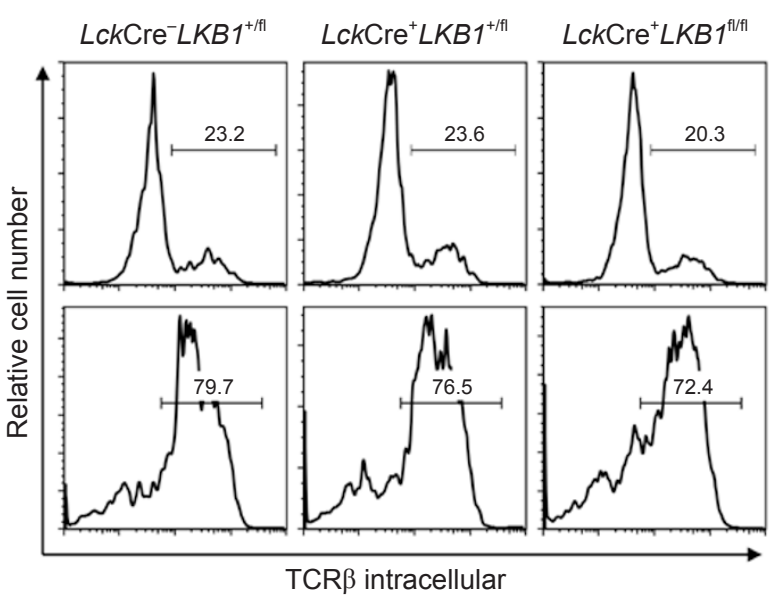

D
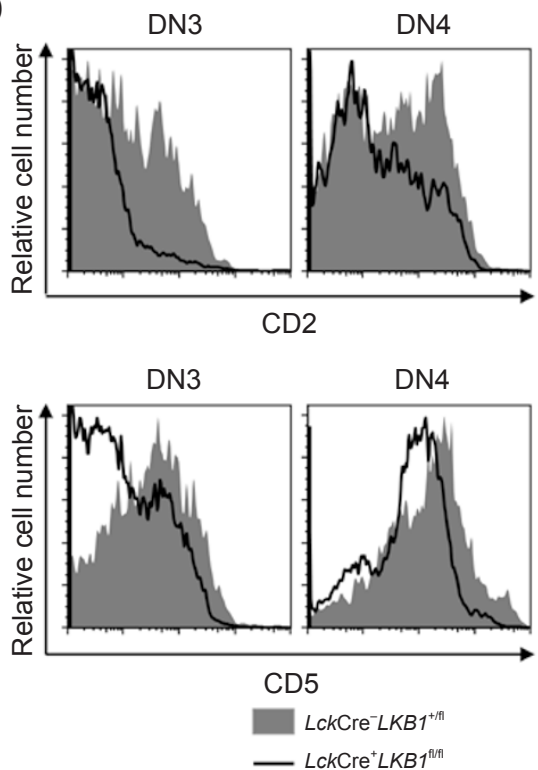

E

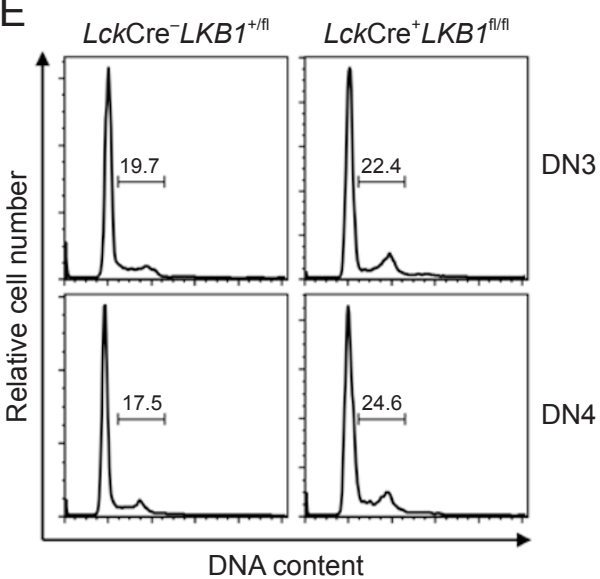

Figure 3 Analysis of DN thymocytes from LKB1-deficient mice. (A) Thymocytes were stained with antibodies to CD4, CD8, CD44 and CD25. The CD44 versus CD25 profiles of gated DN subsets are presented; numbers in quadrants indicate the percentage of cells. (B) Cell numbers of DN subpopulations are calculated as described in the legend to Figure $2 \mathrm{C}$ and indicated as mean \pm SEM. ${ }^{*} P<0.05 ; * * P 0.01$. (C) Thymocytes of indicated genotypes were stained with antibodies to CD4, CD8, CD25 and CD44, then permeabilized and stained with antibody against TCR $\beta$. The brackets indicate the subpopulations expressing intracellular TCR $\beta$, and numbers above the brackets indicate the percentage of TCR $\beta$-positive cells. (D) The surface expression of CD2 and CD5 on DN3and DN4 thymocytes was analyzed. Total thymocytes were stained with antibodies to CD4, CD8, TCR $\beta$, CD44 and CD25. Gated TCR 3 -negative DN3 and DN4 thymocytes were then analyzed for surface protein expression. (E) FACS-sorted DN3 and DN4 thymocytes were permeabilized and incubated in solution containing propidium iodide and RNase. Histograms show the relative DNA content among the indicated DN subsets and numbers above the brackets indicate the percentage of thymocytes in the $S$ and G2/M-phase of the cell cycle. Data are representative of three (A, B, C, D) or four (E) independent experiments. 
chain as their respective controls (Figure 3C). We then asked whether these LKB1-deficient thymocytes could generate efficient pre-TCR signals. To assess this, we examined DN cells for the surface expression of CD2 and CD5 molecules, because the expression levels of these two molecular markers reflect the extent or intensity of pre-TCR signal $[28,29]$. LKB1-deficient DN3 and DN4 thymocytes had lower expression levels of CD2 and CD5 than their wild-type counterparts, respectively (Figure $3 \mathrm{D})$. Given that these cells expressed TCR $\beta$ proteins, LKB1 is thus likely required for efficient pre-TCR signaling, and attenuated pre-TCR signaling might well be a reason for the impaired transition from DN3 to DN4 stage in the absence of LKB1.

We reasoned that the attenuated pre-TCR signaling in LKB1-deficient mice could result in impaired cell proliferation and thereby contribute to the reduced thymic cellularity. However, the percentage of proliferative $(\mathrm{S}+\mathrm{G} 2 /$ M) DN3 and DN4 cells from LKB1-deficient mice were slightly higher than those of their control counterparts from wild-type mice (Figure 3E). These observations were somewhat unexpected, but were in agreement with the proposed role of LKB1 as a tumor suppressor [20, 23].

LKB1-deficient DP thymocytes display accelerated apoptosis

Given that LKB1-deficient mice had almost the same DN cellularity as their littermate controls, we then reasoned that reduced thymic cellularity in LKB1-deficient mice could result from increased cell death of DP thymocytes. To analyze the impact of LKB1 disruption on cell survival, thymocytes were stained with annexin V, a ligand of phosphatidylserine that is expressed on the surface of apoptotic cells [30]. The percentage of annexin V-positive cells in LKB1-deficient DP thymocytes was found higher than that of their respective wild-type counterparts (Figure 4A). In vitro culture experiments were then performed and they revealed that the life span of LKB1-deficient thymocytes was shorter than that of control cells (Figure 4B). These observations indicate that LKB1 is required for DP thymocyte survival.

LKB1-deficient DP thymocytes fail to sustain the expression of Bcl-XL

The anti-apoptotic protein Bcl-XL is specifically expressed in DP thymocytes and contributes to their survival [31]. Consistent with their accelerated death rate, LKB1-deficient DP thymocytes failed to sustain the normal transcription of the Bcl2ll gene (encoding Bcl$\mathrm{XL}$ ); moreover, the transcription of the orphan nuclear receptor gene Rorc (encoding ROR $\gamma \mathrm{t}$, a nuclear factor
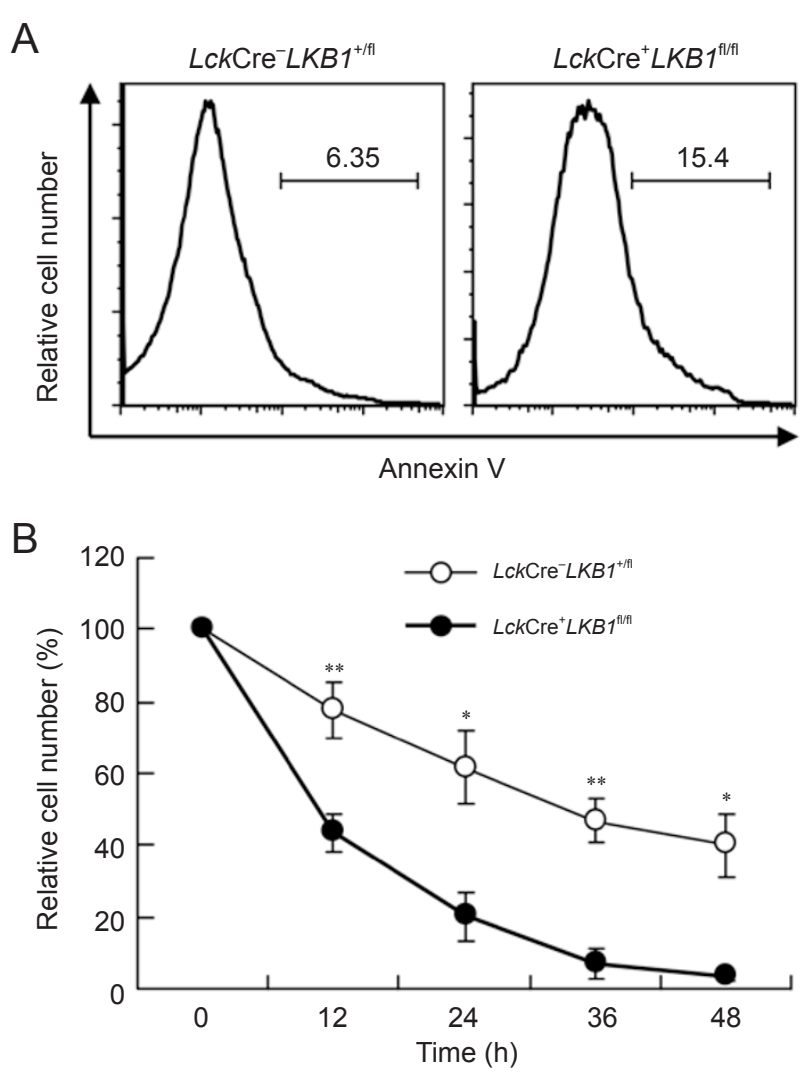

Figure 4 Disruption of LKB1 shortens the survival of DP thymocyte. (A) Thymocytes were surface stained with annexin $V$ in combination with antibodies to CD4 and CD8. Histograms show the percentage of annexin V-positive cells in DP thymocyte subsets. (B) Thymocytes were cultured for the indicated periods of time. At each time point, the percentage of viable thymocytes was determined by negative staining for CD4, CD8 and annexin $\mathrm{V}$ in combination with propidium iodide. Relative survival was calculated as the ratio of surviving cells relative to the onset of the culture. Data are indicated as mean \pm SEM. $* P<0.05$; ** $P$ $<0.01$. Data are representative of three $(B)$ or four $(A)$ independent experiments.

that enhances Bcl-XL expression and promotes thymocyte survival) was also impaired in the absence of LKB1 (Figure 5A).

Based on the above observations, we were curious whether ectopic expression of Bcl-XL could rescue DP thymocytes from LKB1 deficiency-induced cell death. Thymocytes were transfected with the plasmid encoding green fluorescent protein (GFP)-tagged Bcl-XL (GFP$\mathrm{Bcl}-\mathrm{XL}$ ) by electroporation after verifying that the addition of GFP did not alter thymocyte survival (Supplementary information, Figure S1). As shown in Figure $5 \mathrm{~B}$ and $5 \mathrm{C}$, ectopic expression of Bcl-XL efficiently extended the survival of LKB1-deficient DP thymocytes. 
A
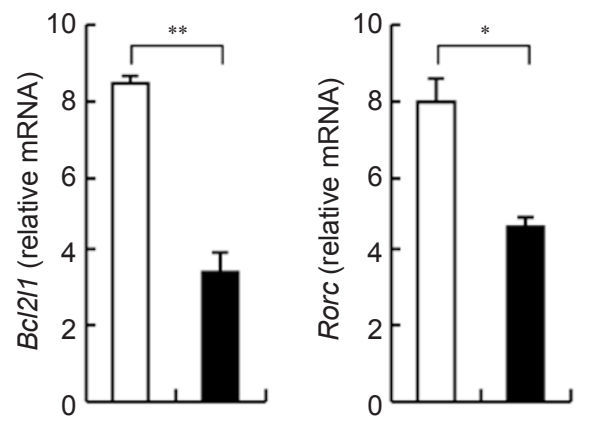

$\square$ LckCre $-L K B 1^{+/ f f}$

$L c k C r e^{+} L K B 1^{\text {furf }}$

B
C

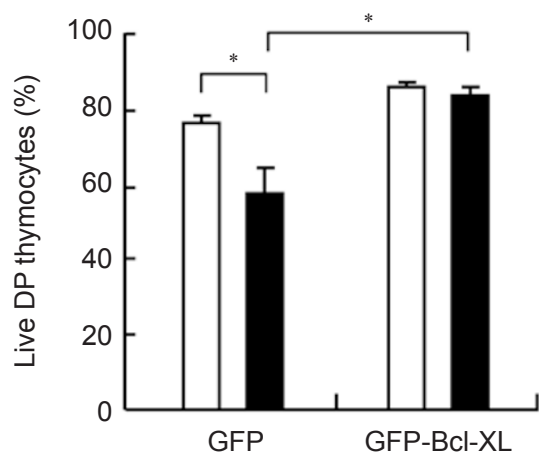

LcKCre- $L K B 1^{+ \text {th }}$

$L c k C r e^{+} L K B 1^{1 / / f}$

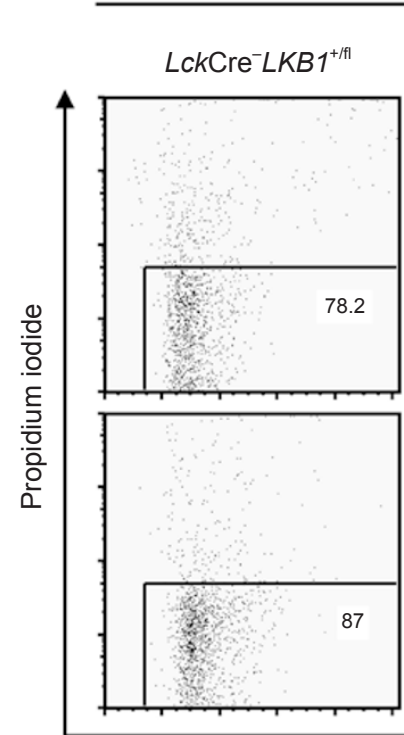

$18 \mathrm{~h}$

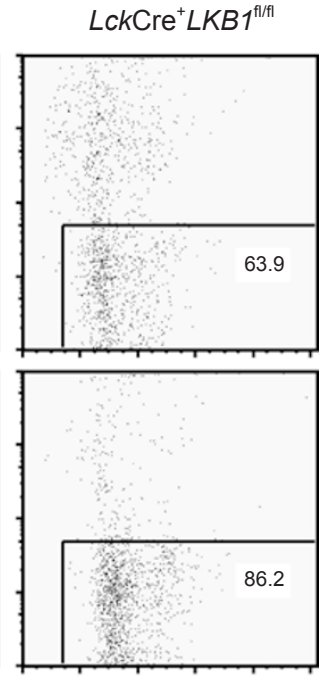

Forward light scatter
$24 \mathrm{~h}$

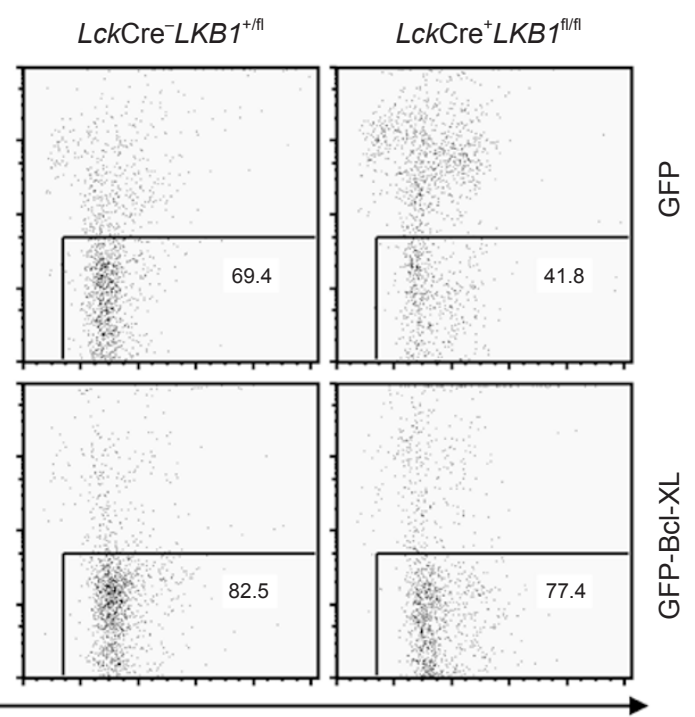

인

$\vec{X}$
$\frac{1}{0}$
0
0
$\frac{1}{4}$
$\frac{1}{0}$

Figure 5 LKB1 deficiency impairs the expression of Bcl-XL and ROR $\gamma \mathrm{t}$. (A) Real-time RT-PCR was used to compare the relative mRNA expression of anti-apoptotic proteins Bcl-XL and ROR $\gamma$ t. Only cDNA encoding ROR $\gamma$ t, but not ROR $\gamma$, was amplified. Each mRNA level was normalized to Hprt mRNA level according to the Ct value and primer efficiency. Results are indicated as mean \pm SEM. ${ }^{*} P<0.05$; ${ }^{* *} P<0.01$. (B) Thymocytes were transfected with plasmids expressing GFP or GFP-Bcl$\mathrm{XL}$ and then cultured for 18 or $24 \mathrm{~h}$, respectively. Cell death of the GFP-positive DP thymocytes was measured by propidium iodide uptake. (C) Relative survival of DP thymocytes was calculated as the ratio of surviving cells relative to the onset of the culture and indicated as mean \pm SEM. $* P<0.05$. Data are representative of three $(\mathbf{B}, \mathbf{C})$ or four $(\mathbf{A})$ independent experiments.

\section{LKB1 controls DP thymocyte survival through activation of $A M P K$}

Considering that AMPK activity affects cell survival $[16,32,33]$, we wondered whether LKB1 deficiency-induced thymocyte death is due to defective AMPK activation. Given that phosphorylation of Thr-172 of AMPK is necessary for its activity [34], the phosphorylation status of AMPK in freshly prepared thymocytes and sorted DP thymocytes was measured. The LKB1-deficient thymocytes showed a reproducible two to threefold decrease in AMPK phosphorylation relative to their controls (Figure 6A). Previous analyses had shown that depletion of the intracellular ATP pool by treatment with the glucose analog, 2-deoxyglucose, normally results in a rapid increase in AMPK phosphorylation [35]. Although this was the case in wild-type DP thymocytes, 2-deoxyglucose failed 
to increase AMPK phosphorylation in LKB1-deficient DP thymocytes (Figure 6B), suggesting that LKB1 is required for AMPK activation in thymocytes on energy stress.

A

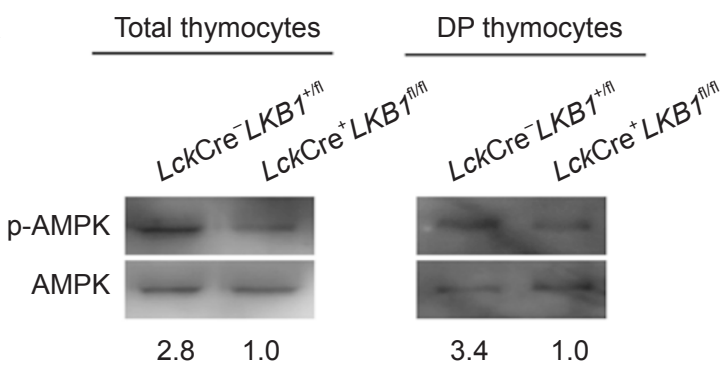

B

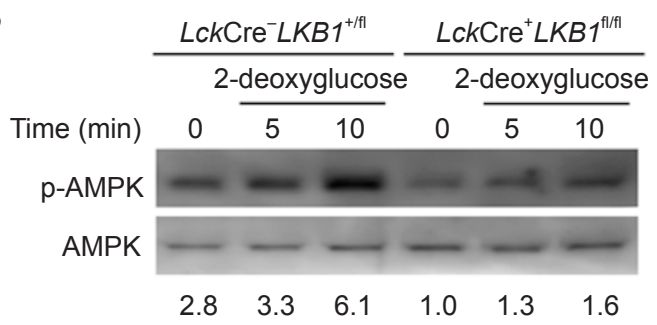

C

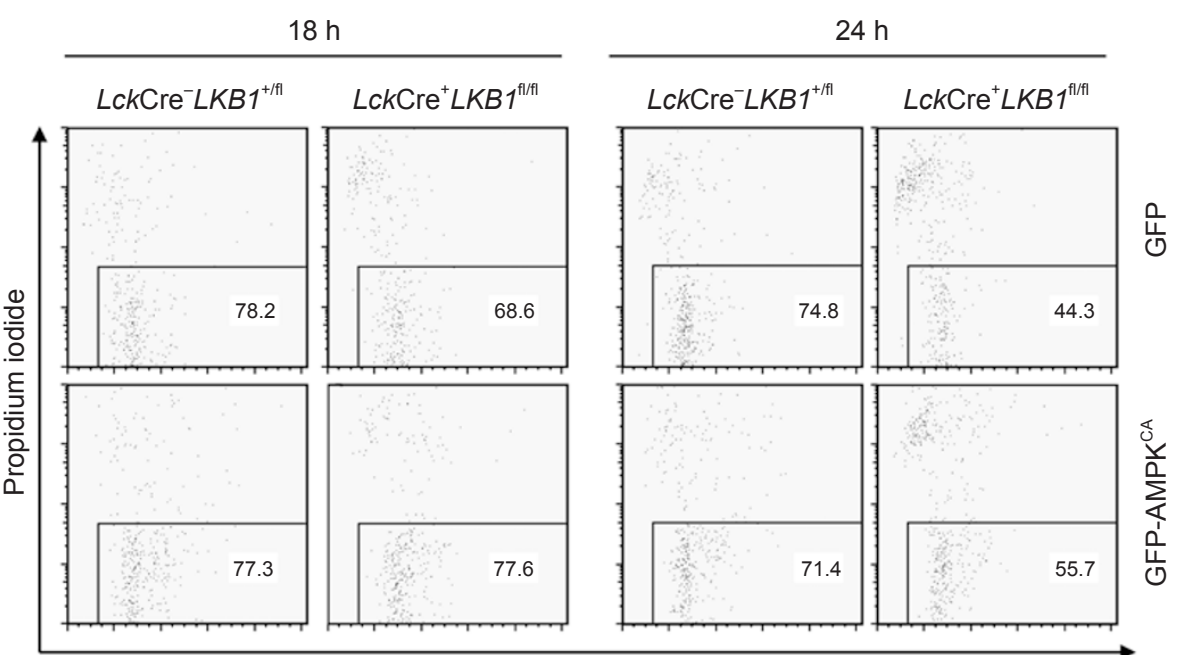

Forward light scatter

\section{$\stackrel{0}{\stackrel{0}{0}}$}

$\sum_{\substack{1 \\ \frac{1}{1}}}^{\frac{1}{0}}$
We then asked whether ectopic expression of the constitutively active AMPK mutant $\left(\mathrm{AMPK}^{\mathrm{CA}}\right)$, a recombinant $\mathrm{AMPK} \alpha 2$ protein that contains a T172D mutation and is truncated at residue 312 [34], could rescue DP thy-

$\mathrm{D}$

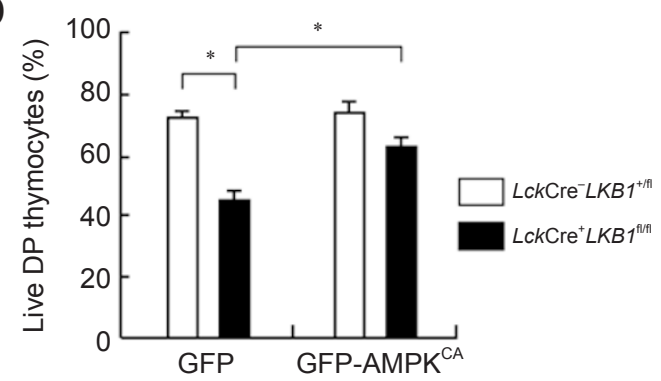

$E$

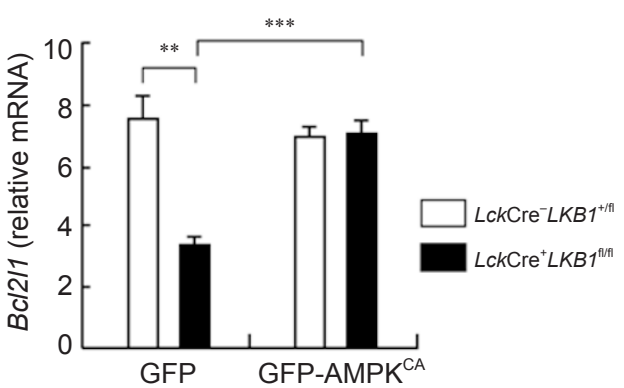

Figure 6 Regulation of DP thymocyte survival by LKB1/AMPK pathway. (A) Cell lysates of freshly prepared total thymocytes (Left) or sorted DP thymocytes (Right) were resolved by SDS-PAGE and analyzed by western blotting with antibodies against phosphor-Thr-172-AMPK $\alpha$ (p-AMPK) and AMPK $\alpha$ (AMPK). The numbers underneath the bands refer to the relative stimulation of AMPKa phosphorylation. (B) Western blot analysis of total cell lysates prepared from 2-deoxyglucose-treated cells (5 or $10 \mathrm{~min}$ ) or untreated thymocytes $(0 \mathrm{~min})$ with phosphor-Thr-172-AMPK $\alpha$ and AMPK $\alpha$ antibodies. The numbers underneath the bands refer to the relative stimulation of AMPK $\alpha$ phosphorylation. (C) Thymocytes were transfected with plasmids expressing GFP or GFP-AMPK ${ }^{\mathrm{CA}}$ and then cultured for 18 or $24 \mathrm{~h}$, respectively. Cell death of the GFP-positive DP thymocytes was measured by propidium iodide uptake. (D) Relative survival of DP thymocytes at $24 \mathrm{~h}$ was calculated as described in the legend to Figure $5 \mathrm{C}$ and indicated as mean \pm SEM. $* P<0.05$. (E) The mRNA level of Bcl-XL of thymocytes transfected with plasmids expressing GFP or GFP-AMPK ${ }^{\mathrm{CA}}$ after $24 \mathrm{~h}$ was measured by real-time RT-PCR. Results are presented as mean \pm SEM. ${ }^{*} P<0.01$; $* * * P<0.001 n=3$. Data are representative of three (A, B, E) or four (C, D) independent experiments. 
mocytes from LKB1 deficiency-induced cell death. To this end, thymocytes were transfected with the plasmid encoding GFP-tagged AMPK $^{\mathrm{CA}}\left(\mathrm{GFP}^{-} \mathrm{AMPK}^{\mathrm{CA}}\right)$ by electroporation. Ectopic expression of GFP-AMPK ${ }^{\mathrm{CA}}$ but not of GFP efficiently promoted cell survival (Figure 6C and 6D). Importantly, ectopic expression of GFP-AMPK ${ }^{\mathrm{CA}}$ also promoted Bcl-XL expression in LKB1-deficient DP thymocytes (Figure 6E). These results demonstrate that LKB1 is required for thymocyte survival, and this effect is, at least in part, mediated by its activation of AMPK and upregulation of Bcl-XL.

\section{Discussion}

The Ser/Thr kinase LKB1 mainly acts as a bona fide tumor suppressor that is involved in the regulation of cell growth, proliferation and polarity $[15,19]$. In this study, we identified LKB1 as an essential regulator of intrathymic T-cell development. A very recent publication from the Cantrell lab also reported that deletion of LKB1 prevents T-cell development [36]. Both studies showed that LckCre-mediated deletion of LKB1 in T cells led to reduced thymus size, blocked transition from DN3 to DN4 stage and impaired generation of CD4 SP and CD8 SP thymocytes. The main phenotypic difference is that the mice used in our study had more DP thymocytes than the mice used in their study, even though both kinds of LKB1-deficient mice had significantly reduced number of DP thymocytes than their control littermates. The phenotypic difference between the two studies might be caused by different expression pattern of the Lck promoter-driven Cre recombinase. Nevertheless, these LKB1-deficient DP thymocytes provide us an opportunity to study the molecular mechanisms by which LKB1 controls the survival of these cells.

Survival is one of the most important events in thymocyte development, and thymocyte survival greatly depends on extracellular signals $[1,2]$. Since DP thymocytes express the anti-apoptotic molecule $\mathrm{Bcl}-2$ and the trophic factors such as glucose transporter Glut1 and IL$7 \mathrm{R}$ at a low level $[1,2,31]$, short-lived DP thymocytes must efficiently maintain the cellular energy homeostasis and thereby extend their life span to be positively selected $[1,2]$. However, the molecular mechanisms that mediate this process remain largely unknown. Interestingly, wild-type DP thymocytes express LKB1 at a high level and maintain basal phosphorylation of AMPK, which reflects that a substantial degree of AMPK activation is required for normal energy balance of DP thymocytes. LKB1-deficient DP thymocytes failed to do so, and moreover, they were unable to immediately elevate AMPK phosphorylation under energy depletion condi- tions. Interestingly, ectopic expression of the constitutively active form of AMPK $\alpha 2$ successfully rescued DP thymocytes from LKB1 deficiency-induced cell death. Notably, previous study showed that AMPK $\alpha 1$ deficiency does not affect thymocyte survival and development [37]. Considering that LKB1 is able to phosphorylate the $\mathrm{T}$ loop of at least 14 related protein kinases that belong to the AMPK subfamily [15], it is possible that AMPK $\alpha 1$ deficiency may be compensated by other members of the AMPK subfamily. Taken together, LKB1 is critical for DP thymocytes to activate AMPK and extend their life span.

Bcl-XL is expressed in DP but not in DN or SP thymocytes, and plays a critical role in protecting DP thymocytes from death by neglect [38], while the orphan receptor ROR $\gamma t$ could prevent the death of premature thymocytes via upregulation of Bcl-XL [39]. LKB1deficient DP thymocytes failed to sustain the expression of Bcl-XL and ROR $\gamma \mathrm{t}$, and ectopic expression of Bcl-XL dramatically promoted the survival of LKB1-deficient DP thymocytes, implying that LKB1 deficiency-induced accelerated cell death is due to defect in maintaining the expression of Bcl-XL. Furthermore, ectopic expression of the constitutively active form of AMPK $\alpha 2$ restored the expression of $\mathrm{Bcl}-\mathrm{XL}$ and at least partially rescued the survival of LKB1-deficient DP thymocytes. Thus, our results identify Bcl-XL as a novel downstream target of LKB1/AMPK signaling.

Defect in survival of DP thymocytes could be a main reason why LKB1-deficient mice failed to generate sufficient CD4 SP and CD8 SP thymocytes. Whether other defects also contribute to this failure remains to be defined. Of note, LKB1 heterozygous mice had $20 \%$ fewer CD4 and CD8 T cells than did wild-type mice in the periphery. Given that haploinsufficiency of LKB1 can cause dominantly inherited cancer $[15,19,23,40]$, it would be of interest to investigate the relationship of host immune status and tumor development when LKB1 activity is aberrant.

\section{Materials and Methods}

\section{Mice}

The $L K B 1$ floxed alleles [20], as well as the $L c k C r e$ [26] mouse strains, have been described previously. $L c k$ Cre transgenic mice were crossed with $L K B 1^{f / f l}$ mice to generate $L c k \mathrm{Cre}^{+} L K B 1^{\mathrm{f} / \mathrm{fl}}$ (carrying one $L c k$ Cre transgene and two floxed alleles, homozygous mutant), $L c k \mathrm{Cre}^{-} L K B I^{+/ f 1}$ (carrying one floxed allele, wild-type control) and $L c k \mathrm{Cre}^{+} L K B 1^{+/ f l}$ (carrying $L c k C r e$ transgene, one wild-type and one floxed allele, heterozygous mutant) mice. Mice were analyzed between 4- and 8-weeks of age. All mice were maintained in SPF facility and were genotyped by PCR analysis with genomic DNA before use. A few LKB1-deficient mice were 
observed with leaky deletion of LKB1 beyond DN4 stage, and this kind of mouse was not used for further analysis in this study ( LckCre-related leaky deletion has been described by Orkin and his co-workers [41]). All animal protocols and experiments were approved by the institutional animal use committee of the Shanghai Institutes for Biological Sciences, Chinese Academy of Sciences.

\section{Antibodies}

The following monoclonal antibodies used for staining and cell sorting were from BD Pharmingen: anti-CD4 (GK1.5 and RM44), anti-CD8 $\alpha$ (53-6.7), anti-TCR $\beta$ (H57-597), anti-CD44 (IM-7), anti-CD25 (3C7), anti-CD5 (53-7.3) and anti-CD2 (RM2-5). Antiphospho-AMPK $\alpha$ (40H9) and anti-AMPK $\alpha$ (23A3) were bought from Cell Signaling Technology.

\section{Cell preparation, staining and purification}

Single-cell thymocyte or lymphocyte suspensions were prepared and surface stained as described [11, 42]. Cell fluorescence was acquired on a two-laser FACSCalibur (BD Biosciences) and was analyzed with FlowJo software. The intracellular staining was done as described before [43]. DN and DP thymocytes were sorted by a FACSAria II (BD Biosciences) after surface stained with antiCD4 and anti-CD8 antibodies. DN1-4 subsets were purified by cell sorting after magnetic depletion of DP, CD4 SP and CD8 SP thymocytes (Miltenyi Biotec). Cell purity assessed by surface staining and flow cytometry was over $90 \%$.

\section{Cell cycle analysis}

Cell cycle analysis was done as described [44]. Sorted DN3 and DN4 thymocytes were fixed by $70 \%$ ethanol, digested by RNase (200 $\mu \mathrm{g} / \mathrm{ml}$, Sigma), stained by propidium iodide $(5 \mu \mathrm{g} / \mathrm{ml}$, Sigma) and then analyzed by flow cytometry.

\section{In vitro cell survival assay}

In vitro cell survival assay was done as described [30]. Thymocytes were resuspended at a density of $1 \times 10^{6}$ cells per $\mathrm{ml}$ in complete RPMI 1640 medium, then placed in a cell culture incubator at $37{ }^{\circ} \mathrm{C}$ under $5 \% \mathrm{CO}_{2}$ for $12,24,36$ and $48 \mathrm{~h}$ separately. Thymocytes were stained with annexin $\mathrm{V}$ and propidium iodide (Roche Applied Bioscience) and live cells were gated by exclusion of annexin $\mathrm{V}$ - and propidium iodide-positive cells.

\section{Real-time RT-PCR}

RNA was extracted and quantified as described [42]. RNA was reverse-transcribed to cDNA with SuperScript III First-Strand kit (Invitrogen). The mRNA levels of $L K B 1, B c l 2 l 1$ and RORc were assessed relative to Hprt mRNA level by real-time RT-PCR (Rotor gene 6000; Corbett Life Sciences) with SYBR Green real-time PCR Master Mix (Toyobo Co Ltd). The data showed were relative values. Primers are listed in Supplementary information, Table S1.

\section{Electroporation transfection}

Thymocytes $\left(10 \times 10^{6}\right)$ were suspended in $250 \mu$ RPMI 1640 medium containing 5\% FBS and 5\% FCS without antibiotics and mixed with plasmids encoding GFP, GFP-Bcl-XL or GFP-AMPK${ }^{\mathrm{CA}}$. Electroporation was done in a square wave electroporator (Gene pulser Xcell, Bio-Rad Laboratories) using the machine settings as described [45]: pulse length $=20 \mathrm{~ms}$, pulses $=1,4 \mathrm{~mm}$ Gap cuvettes and voltage $=360 \mathrm{~V}$. Cells were harvested and analyzed at 18 and $24 \mathrm{~h}$.

\section{Statistics}

All experiments showed were performed three times or more. The arithmetic means were calculated and shown as bar graphs and fold-line graphs with error bars of standard error of mean (SEM). Student's $t$-test was used for the comparison of two independent groups.

\section{Acknowledgments}

We thank X Wu (Fudan University) for LckCre mouse and $\mathrm{K}$ Wong (Dana-Farber Cancer Institute) for $L K B 1^{f / f}$ mouse, R Bosselut (National Institutes of Health) and D Li (Shanghai Institutes for Biological Sciences) for instructive comments on the manuscript; We are grateful to our colleagues F Liu for animal husbandry, W Bian for cell sorting and X Wang for real-time PCR analysis. This research was supported in part by the National Natural Science Foundation of China (30872290, 30925031), the Ministry of Science and Technology (2006CB504303, 2007CB815802, 2009ZX10004-105), the Hi-Tech Research and Development Program of China (2007AA02Z167), the National Basic Research Program of China (2007CB914504) and the Chinese Academy of Sciences (KSCX1-YW-R-43, KSCX2-YW-R-10).

\section{References}

1 Fox CJ, Hammerman PS, Thompson CB. Fuel feeds function: energy metabolism and the T-cell response. Nat Rev Immunol 2005; 5:844-852.

2 Plas DR, Rathmell JC, Thompson CB. Homeostatic control of lymphocyte survival: potential origins and implications. Nat Immunol 2002; 3:515-521.

3 Frauwirth KA, Thompson CB. Regulation of T lymphocyte metabolism. J Immunol 2004; 172:4661-4665.

4 Wullschleger S, Loewith R, Hall MN. TOR signaling in growth and metabolism. Cell 2006; 124:471-484.

5 Wolfer A, Wilson A, Nemir M, MacDonald HR, Radtke F. Inactivation of Notch1 impairs VDJbeta rearrengement and allows pre-TCR-independent survival of early alpha beta lineage thymocytes. Immunity 2002; 16:869-879.

6 Ciofani M, Zúñiga-Pflücker JC. Notch promotes survival of pre-T cells at the beta-selection checkpoint by regulating cellular metabolism. Nat Immunol 2005; 6:881-888.

7 Kim K, Lee C-k, Sayers TJ, Muegge K, Durum SK. The trophic action of IL-7 on pro-T cells: inhibition of apoptosis of pro-T1, -T2, and -T3 cells correlates with Bcl-2 and Bax levels and is independent of Fas and p53 pathways. J Immunol 1998; 160:5735-5741.

8 Michie AM, Zúñiga-Pflücker JC. Regulation of thymocyte differentiation: pre-TCR signals and beta-selection. Semin Immunol 2002; 14:311-323.

9 Linette GP, Grusby MJ, Hedrick SM, Hansen TH, Glimcher $\mathrm{LH}$, Korsmeyer SJ. Bcl-2 is upregulated at the $\mathrm{CD} 4^{+} \mathrm{CD} 8^{+}$ stage during positive selection and promotes thymocyte differentiation at several control Points. Immunity 1994; 1:197205.

10 Williams O, Norton T, Halligey M, Kioussis D, Brady HJM. The action of Bax and Bcl-2 on T cell selection. J Exp Med 1998; 188:1125-1133.

11 Wang X, Zhang Y, Xiao G, Gao X, Liu X. c-Fos enhances the survival of thymocytes during positive selection by upregulat- 
ing Bcl-2. Cell Res 2008; 19:340-347.

12 Huang EY, Gallegos AM, Richards SM, Lehar SM, Bevan MJ. Surface expression of Notch1 on thymocytes: correlation with the double-negative to double-positive transition. $J \mathrm{Im}$ munol 2003; 171:2296-2304.

13 Yu Q, Erman B, Park J-H, Feigenbaum L, Singer A. IL-7 receptor signals inhibit expression of transcription factors TCF1, LEF-1, and RORgammat: impact on thymocyte development. J Exp Med 2004; 200:797-803.

14 Mombaerts P, Clarke AR, Rudnicki MA, et al. Mutations in T-cell antigen receptor genes alpha and beta block thymocyte development at different stages. Nature 1992; 360:225-231.

15 Alessi DR, Sakamoto K, Bayascas JR. LKB1-dependent signaling pathways. Annu Rev Biochem 2006; 75:137-163.

16 Shaw RJ, Kosmatka M, Bardeesy N, et al. The tumor suppressor LKB1 kinase directly activates AMP-activated kinase and regulates apoptosis in response to energy stress. Proc Natl Acad Sci USA 2004; 101:3329-3335.

17 Woods A, Johnstone SR, Dickerson K, et al. LKB1 is the upstream kinase in the AMP-activated protein kinase cascade. Curr Biol 2003; 13:2004-2008.

18 Carling D. The AMP-activated protein kinase cascade - a unifying system for energy control. Trends Biochem Sci 2004; 29:18-24.

19 Spicer J, Ashworth A. LKB1 kinase: master and commander of metabolism and polarity. Curr Biol 2004; 14:R383-R385.

20 Bardeesy N, Sinha M, Hezel AF, et al. Loss of the Lkb1 tumour suppressor provokes intestinal polyposis but resistance to transformation. Nature 2002; 419:162-167.

21 Jishage K, Nezu J, Kawase Y, et al. Role of Lkb1, the causative gene of Peutz-Jegher's syndrome, in embryogenesis and polyposis. Proc Natl Acad Sci USA 2002; 99:8903-8908.

22 Hemminki A, Markie D, Tomlinson I, et al. A serine/threonine kinase gene defective in Peutz-Jeghers syndrome. Nature 1998; 391:184-187.

23 Miyoshi H, Nakau M, Ishikawa TO, Seldin MF, Oshima M, Taketo MM. Gastrointestinal hamartomatous polyposis in Lkb1 heterozygous knockout mice. Cancer Res 2002; 62:2261-2266.

24 Ossipova O, Bardeesy N, DePinho RA, Green JBA. LKB1 (XEEK1) regulates Wnt signalling in vertebrate development. Nat Cell Biol 2003; 5:889-894.

25 Ylikorkala A, Rossi DJ, Korsisaari N, et al. Vascular abnormalities and deregulation of VEGF in Lkb1-deficient mice. Science 2001; 293:1323-1326.

26 Pan L, Hanrahan J, Li J, Hale LP, Zhuang Y. An analysis of T cell intrinsic roles of E2A by conditional gene disruption in the thymus. J Immunol 2002; 168:3923-3932.

27 Godfrey DI, Kennedy J, Suda T, Zlotnik A. A developmental pathway involving four phenotypically and functionally distinct subsets of $\mathrm{CD}^{-} \mathrm{CD} 4^{-} \mathrm{CD} 8^{-}$triple-negative adult mouse thymocytes defined by CD44 and CD25 expression. J Immunol 1993; 150:4244-4252.

28 Rodewald HR, Awad K, Moingeon P, et al. Fc gamma RII/ III and CD2 expression mark distinct subpopulations of immature CD4CD8 murine thymocytes: in vivo developmental kinetics and $\mathrm{T}$ cell receptor beta chain rearrangement status. $J$
Exp Med 1993; 177:1079-1092.

29 Azzam HS, Grinberg A, Lui K, Shen H, Shores EW, Love PE. CD5 expression is developmentally regulated by $\mathrm{T}$ cell receptor (TCR) signals and TCR avidity. J Exp Med 1998; 188:2301-2311.

30 Goux D, Coudert JD, Maurice D, et al. Cooperating pre-Tcell receptor and TCF-1-dependent signals ensure thymocyte survival. Blood 2005; 106:1726-1733.

31 Chao DT, Korsmeyer SJ. Bcl-2 family: regulators of cell death. Annu Rev Immunol 1998; 16:395-419.

32 Kato K, Ogura T, Kishimoto A, et al. Critical roles of AMPactivated protein kinase in constitutive tolerance of cancer cells to nutrient deprivation and tumor formation. Oncogene 2002; 21:6082-6090.

33 Luo Z, Saha AK, Xiang X, Ruderman NB. AMPK, the metabolic syndrome and cancer. Trends Pharmacol Sci 2005; 26:69-76

34 Jones RG, Plas DR, Kubek S, et al. AMP-activated protein kinase induces a p53-dependent metabolic checkpoint. Mol Cell 2005; 18:283-293.

35 Tamas P, Hawley SA, Clarke RG, et al. Regulation of the energy sensor AMP-activated protein kinase by antigen receptor and $\mathrm{Ca}^{2+}$ in T lymphocytes. J Exp Med 2006; 203:1665-1670.

36 Tamás P, Macintyre A, Finlay D, et al. LKB1 is essential for the proliferation of $\mathrm{T}$ cell progenitors and mature peripheral $\mathrm{T}$ cells. Eur J Immunol 2009; 9999:NA.

37 Mayer A, Denanglaire S, Viollet B, Leo O, Andris F. AMPactivated protein kinase regulates lymphocyte responses to metabolic stress but is largely dispensable for immune cell development and function. Eur J Immunol 2008; 38:948-956.

38 Ma A, Pena JC, Chang B, et al. Bclx regulates the survival of double-positive thymocytes. Proc Natl Acad Sci USA 1995; 92:4763-4767.

39 Sun Z, Unutmaz D, Zou Y-R, et al. Requirement for RORgamma in thymocyte survival and lymphoid organ development. Science 2000; 288:2369-2373.

40 Yoo LI, Chung DC, Yuan J. LKB1 -- A master tumour suppressor of the small intestine and beyond. Nat Rev Cancer 2002; 2:529-535.

41 Mao X, Fujiwara Y, Orkin SH. Improved reporter strain for monitoring Cre recombinase-mediated DNA excisions in mice. Proc Natl Acad Sci USA 1999; 96:5037-5042.

42 Wang X, Xiao G, Zhang Y, et al. Regulation of Tcrb recombination ordering by c-Fos-dependent RAG deposition. Nat Immunol 2008; 9:794-801.

43 Liu X, Adams A, Wildt KF, Aronow B, Feigenbaum L, Bosselut R. Restricting Zap70 expression to $\mathrm{CD} 4^{+} \mathrm{CD} 8^{+}$thymocytes reveals a $\mathrm{T}$ cell receptor-dependent proofreading mechanism controlling the completion of positive selection. J Exp Med 2003; 197:363-373.

44 Hock H, Hamblen MJ, Rooke HM, et al. Gfi-1 restricts proliferation and preserves functional integrity of haematopoietic stem cells. Nature 2004; 431:1002-1007.

45 Bell MP, Huntoon CJ, Graham D, McKean DJ. The analysis of costimulatory receptor signaling cascades in normal $\mathrm{T}$ lymphocytes using in vitro gene transfer and reporter gene analysis. Nat Med 2001; 7:1155-1158.

(Supplementary information is linked to the online version of the paper on the Cell Research website.) 